\title{
INTERVENCIÓN PSICOTERAPÉUTICA GRUPAL INTENSIVA REALIZADA EN UN ENTORNO NATURAL PARA EL TRATAMIENTO DEL BURNOUT EN UN EQUIPO DE ENFERMERÍA ONCOLÓGICA
}

\section{INTENSIVE GROUP PSYCHOTHERAPY INTERVENTION IN A NATURAL ENVIRONMENT FOR THE TREATMENT OF BURNOUT IN AN ONCOLOGY NURSING TEAM}

\author{
Jerónima Vázquez-Ortizi,2, Ma Jesús Vázquez-Ramos' , Marta Álvarez-Serranol, \\ María Reyes-Alcaide', Rosario Martínez-Domingo', Marta Nadal-Delgado' \\ y Juan Antonio Cruzado 3 \\ ' Asociación Oncológica Extremeña (AOEx) \\ 2 Centro Universitario Santa Ana (Almendralejo) \\ ${ }^{3}$ Facultad de Psicología, Universidad Complutense de Madrid
}

Resumen

Este estudio piloto tiene como objetivo valorar la influencia que tiene la intervención psicoterapéutica grupal sobre el estrés y el burnout en un equipo de enfermería oncológica. La intervención psicológica consistió en la aplicación de un paquete para la inoculación al estrés, utilizando principalmente técnicas cognitivo-conductuales, Ilevándose a cabo de forma intensiva, a través de la convivencia del grupo durante tres días en un alojamiento situado en un entorno natural de montaña.

Utilizamos un diseño cuasi-experimental de dos grupos (experimental y control) con medidas pre y post-tratamiento. El grupo experimental estaba compuesto por los nueve miembros del equipo de enfermería que recibió el tratamiento psicológico y el grupo control estuvo compuesto por once profesionales de otros equipos de enfermería oncológica. Las variables dependientes consideradas fueron, de un lado, ansiedad y depresión (evaluadas con la Escala Hospitalaria de Ansiedad y Depresión) y, de otro lado, cansancio emocional, despersonalización y realización personal (evaluadas con el Maslach Burnout Inventory).

Los resultados muestran que transcurrido un mes de la intervención psicoterapéu-

\section{Abstract}

This pilot study aims to test the influence of a group psychotherapeutic intervention on stress and burnout in an oncology nursing team. The psychological intervention consisted of implementing a package for stress inoculation, mainly using cognitive-behavioral techniques. The intervention is carried out intensively, through the coexistence of the group for three days in an accommodation located in a natural mountain environment.

We used a quasi-experimental design of two groups (experimental and control) with pre and post-treatment measures. The experimental group was composed of nine members of the nursing team that received the psychological treatment and the control group was composed of eleven professionals from other teams of oncology nursing. The dependent variables considered were, on one hand anxiety and depression (evaluated by the Hospital Anxiety and Depression Scale) and, on the other hand, emotional exhaustion, depersonalization, and personal fulfilment (evaluated by the Maslach Burnout Inventory).

The experimental group improved significantly in anxiety $(Z=-2.44, P=0.015$, 
tica grupal, en el grupo de tratamiento se había producido una mejoría significativa de la ansiedad $(Z=-2,44 ; p=0,015 ; d=0,71)$ y de la realización personal $(Z=-2,54 ; \quad p=0,011$; $d=-1,29)$, igualmente se produjo en este período un gran efecto positivo sobre el cansancio emocional $(Z=-2,08 ; p=0,037 ; d=0,94)$. En el pre-tratamiento el grupo experimental manifestaba mayores signos de desgaste profesional que el grupo control, principalmente en lo referido a la falta de realización personal $(U=14$; $p=0,006 ; d=-1,61)$, de hecho había demandado la intervención psicológica. Tras la experiencia terapéutica se produjo una equiparación en los valores de las variables dependientes consideradas entre los grupos, respecto a la situación pre-tratamiento.

Al año de la intervención, en el grupo tratado sigue habiendo un efecto positivo importante sobre la realización personal $(Z=-2,24$; $\mathrm{p}=0,025 ; d=-1,15)$ y sobre la despersonalización $(Z=-2,23 ; p=0,026 ; d=0,52)$. Concluimos que el tratamiento cognitivo-conductual grupal, intensivo realizado en un medio natural, ajeno al hospital, puede tener notables ventajas para el tratamiento del desgaste profesional en personal de enfermería oncológica.

Palabras clave: Desgaste profesional, estrés laboral, síndrome de burnout, intervención psicoterapéutica, terapia grupal, enfermería oncológica. $d=0.71)$, personal fulfilment $(Z=-2.54$, $P=0.011, d=-1.29)$ and emotional exhaustion $(Z=-2.08 ; \quad p=0.037 ; d=0.94)$ one month post -treatment. In the pre-treatment, The experimental group showed burnout levels higher than the control group, particularly with regard to the lack of personal personal fulfilment $(U=14, P=0.006, d=-1.61)$, mainly regarding the lack of personal fulfillment $(U=14 ; p=0.006 ; d=-1.61)$. in fact the experimental group had requested psychological intervention. In fact, the experimental group had requested psychological intervention. After the therapeutic experience, the values of the dependent variables were equal in both groups.

One year after the intervention, the treated group is still having a significant positive effect on the personal fulfilment $(Z=-2.24 ; p=0.025$; $d=-1.15)$ and on the depersonalization $(Z=-2.23 ; \quad p=0.026 ; d=0.52)$. We conclude that an intensive program of group cognitive behavioral therapy in a natural setting outside the hospital can be an effective strategy of burnout treatment in oncology nurses.

Keywords: Professional burnout, workrelated stress, burnout, psychotherapeutic intervention, group therapy, oncology nursing.

\section{INTRODUCCIÓN}

El desgaste profesional o Síndrome de Burnout se caracteriza ${ }^{(1,2)}$ por la presencia de agotamiento o fatiga emocional, actitudes insensibles o despersonalización en el trato y baja realización personal, con sentimientos de incompetencia, afectando de manera muy especial a profesionales cuyo trabajo exige un contacto directo con otras personas, y donde tienen que dar respuesta a situaciones con altas demandas emocionales, junto con altas dosis de implicación y capacidad de entrega ${ }^{(3,4)}$.

Los profesionales de la salud son más vulnerables al estrés crónico laboral y al desgaste emocional, debido a que su traba- jo les pone en interacción directa con los problemas psicológicos, físicos y/o sociales de los pacientes ${ }^{(5,6)}$. El burnout se considera consecuencia de un proceso crónico de estrés laboral(7), en el que el profesional sanitario utiliza estrategias de afrontamiento que dejan de ser eficaces ante la perpetuación de la situación estresante en el tiempo ${ }^{(8)}$. La respuesta ante este proceso de estrés crónico es afectiva (agotamiento emocional), cognitiva (pérdida de ilusión en el trabajo) y actitudinal (despersonalización) y las consecuencias se dejan sentir tanto a nivel personal e individual, como a nivel organizacional, con disminución de la calidad asistencial ${ }^{(7)}$. Datos recientes ${ }^{(9-11)}$ indican que entre una cuarta y una tercera 
parte de los sanitarios que forman parte de los equipos hospitalarios presentan alguno de los tres indicadores de burnout; en el caso del personal de enfermería, el cansancio emocional puede estar presente casi en la mitad de los trabajadores ${ }^{(9)}$.

En lo que respecta a enfermería oncológica, hay evidencia empírica ${ }^{(12,13)}$ que demuestra la mayor incidencia de estrés laboral y desgaste profesional en esta especialidad en comparación con otras (a excepción de enfermería de $\mathrm{UCl}$, que presenta niveles similares), particularmente en lo relativo al agotamiento emocional y a la baja realización personal. Esta elevada incidencia tiene relación, entre otros factores, con el esfuerzo emocional que tienen que hacer estos profesionales, atendiendo a demandas múltiples, continuas y cambiantes que, en muchos casos, pueden ser contrapuestas entre médicos, pacientes y familiares ${ }^{(12)}$, a lo cual se une la carencia de formación en habilidades de comunicación, que les proporcione herramientas para manejar situaciones difíciles de interacción que de forma continua les pone en contacto con el sufrimiento de las personas ${ }^{(14)}$. Sabemos que una de las repercusiones más habituales del burnout en enfermería oncológica es la intención de abandono de la especialidad ${ }^{(12)}$, con la consecuente pérdida de potencial humano experto en el área.

Dicho todo lo anterior, destacamos la relevancia de la intervención psicológica en burnout, la cual puede tener un carácter preventivo o terapéutico y puede llevarse a cabo a nivel individual o grupal. En cualquier caso, dicha intervención forma parte de la labor del psicooncólogo del propio equipo sanitario (bien en Oncología Médica o bien en Cuidados Paliativos) ${ }^{(15)}$.

En cuanto a las líneas de intervención para el burnout en sanitarios, los trabajos empíricos apuntan ${ }^{(16)}$ hacia la efectividad de estrategias como las habilidades asertivas y de comunicación, la estimulación del trabajo en equipo o la búsqueda de sentido a la acción profesional. No hay una estrategia terapéutica simple que por sí sola resulte efectiva para tratar el desgaste profesional en personal de oncología ${ }^{(17)}$, de ahí la importancia de las intervenciones de tipo multicomponente.

Cancio López y García Llana ${ }^{(16)}$ indican una serie de pautas para el tratamiento del desgaste profesional en personal de oncología que incluye herramientas de trabajo a nivel individual (con técnicas de autorregulación de emociones, cogniciones, reacciones fisiológicas, conductas y valores), a nivel interpersonal (con formación en counselling como herramienta de comunicación) y a nivel organizacional (con la promoción del trabajo en equipo).

Siguiendo esta línea de trabajo, el modelo de intervención que nosotros proponemos en este estudio piloto, es un programa multicomponente estructurado como un paquete de inoculación al estrés, donde se trabajan aspectos individuales, interpersonales y organizacionales. No obstante, nuestro trabajo psicoterapéutico grupal se realiza de forma intensiva, en un contexto de tratamiento distinto al del trabajo, ajeno a donde habitualmente se relacionan y dando a los participantes la oportunidad de interaccionar durante un período de tiempo grande (varios días), que permita conocerse "sin bata", profundizar en la relación mutua y mejorar la cohesión grupal. Además, el contexto medioambiental natural (entorno rural de montaña) que enmarca todo el proceso de nuestra intervención, puede ser de especial interés de cara a la motivación e implicación de los participantes, con lo que creemos que la intervención psicológica puede resultar más efectiva.

\section{OBJETIVO}

El objetivo general de este estudio piloto, es valorar cómo influye la parti- 
cipación en una experiencia terapéutica grupal dirigida al abordaje del estrés laboral sobre los niveles de ansiedad, de depresión y sobre factores emocionales que definen el síndrome de agotamiento profesional (cansancio emocional, despersonalización y falta de realización personal), en personal de enfermería oncológica, mediante un diseño cuasi-experimental con medidas repetidas de grupo de tratamiento y grupo control. La intervención terapéutica llevada a cabo con el grupo experimental se basa en la aplicación de un paquete de tratamiento con técnicas cognitivo conductuales para la inoculación del estrés y implementada de forma continua e intensiva, en un contexto de convivencia grupal y en un entorno natural de montaña.

La hipótesis con la que se trabaja es que el grupo experimental tras participar en la experiencia terapéutica grupal, mejorará significativamente en los niveles de depresión, ansiedad, cansancio emocional, despersonalización y falta de realización personal. Mientras que el grupo control, sin tratamiento, no cambiará significativamente en ninguno de los parámetros anteriores.

\section{MÉTODO}

\section{Diseño}

La estrategia de investigación del presente trabajo corresponde a un diseño cuasi-experimental mixto de dos grupos, uno experimental y otro control, con medidas pre y post-tratamiento.

La variable independiente es el tratamiento o intervención psicológica grupal, con dos valores: presencia y ausencia. De otro lado, las variables dependientes consideradas fueron las siguientes: a) ansiedad, b) depresión, c) cansancio emocional, d) despersonalización y e) realización personal.

\section{Muestra}

El grupo experimental está formado por miembros del equipo de enfermería del Hospital de Día que atiende a los pacientes de la Unidad de Onco-Hematología del Hospital de Llerena (Badajoz), quienes participaron en un taller para el aprendizaje de técnicas de afrontamiento del estrés laboral, y el grupo control está formado por miembros del personal de enfermería de Hospital de Día de otros hospitales de la Comunidad Autónoma Extremeña (Hospital "Campo Arañuelo" de Navalmoral, Hospital "San Pedro de Alcántara" de Cáceres y Hospital "Virgen del Puerto" de Plasencia), con quienes no se ha llevado a cabo ningún tipo de intervención psicológica grupal dirigida al afrontamiento del estrés o a la prevención del burnout ni previamente, ni durante el período de evaluación.

En el grupo experimental participaron los nueve componentes del equipo de enfermería de Hospital de Día primeramente mencionado, siendo ocho mujeres y un hombre. La edad media del grupo era de 47,7 años $(\mathrm{dt}=7,91)$ en el momento del pre-test. En la fecha en que se llevó a cabo el Taller (marzo de 2011), el equipo estaba compuesto por seis enfermeros $(67 \%)$ -cinco enfermeras y un enfermero- y tres auxiliares de enfermería (33\%), todas ellas mujeres. En relación al estado civil de los participantes, decir que 8 (89\%) estaban casados o con pareja de hecho y una de las mujeres estaba soltera (11\%).

En el grupo control participaron once miembros de personal de enfermería oncológica con dedicación a Hospital de Día, todas ellas mujeres, de las cuales seis pertenecen al Hospital "Campo Arañuelo", cuatro al Hospital "San Pedro de Alcántara" y una al Hospital "Virgen del Puerto". El $55 \%$ son enfermeras y el $45 \%$ auxiliares de enfermería. La media de la edad de este grupo es de 47,18, con una desviación tí- 
pica de 6,85. De las 11 mujeres, 9 estaban casadas $(82 \%)$ y 2 estaban separadas o divorciadas $(18 \%)$.

El grupo experimental lleva una media de 21,22 años $(\mathrm{dt}=6,32)$ en la profesión y 7,95 años de media $(\mathrm{dt}=3,86)$ en el puesto de Hospital de Día. Por su parte, el grupo control lleva unos 22,94 años de media $(\mathrm{dt}=8,79)$ en la profesión y 8,54 años de media en Hospital de Día (dt=9,43).

\section{Instrumentos}

Para llevar a cabo el presente trabajo de investigación, hemos utilizado los siguientes instrumentos de evaluación:

El Maslach Burnout Inventory (MBI) de Maslach y Jackson ${ }^{(1)}$, en su versión española ${ }^{(18)}$, que evalúa tres variables del Síndrome del Burnout: Cansancio Emocional, Despersonalización y Realización Personal. El MBI está compuesto por 22 ítems que se puntúan en una escala de 0 a 6 , correspondiente a distintos niveles de frecuencia. La puntuación de cada subescala se calcula sumando la puntuación de los ítems correspondientes a la misma. Son indicadoras de alto nivel de burnout las puntuaciones altas en Cansancio Emocional y en Despersonalización, así como las puntuaciones bajas en Realización Personal. Hemos utilizado los siguientes puntos de corte ${ }^{(19,20)}$ :

- Subescala de Cansancio Emocional (MBI-CE): entre 19 y 26, ambos inclusive, es el nivel medio, siendo por debajo de 19 nivel bajo y por encima de 26 el nivel alto de burnout.

- Subescala de Despersonalización (MBI-DP): entre 6 y 9 , ambos inclusive, es nivel medio, por debajo de 6 es nivel bajo y por encima de 9 es nivel alto de burnout.

- Subescala de Realización Personal (MBI-RP): nivel medio entre $34 \mathrm{y}$ 39, ambos inclusive; nivel bajo de burnout superior a 39 y nivel alto de burnout inferior a 34 .

La Escala Hospitalaria de Ansiedad y Depresión (HAD) de Zigmond y Snaith ${ }^{(21)}$, con adaptación española de Caro e Ibáñez ${ }^{(22)}$, ha sido diseñada para detectar síntomas de depresión y ansiedad durante la semana anterior a la evaluación en población no psiquiátrica. A través de sus dos subescalas, nos proporciona una puntuación de ansiedad (HAD-A) y otra de depresión (HAD-D). La puntuación varía de 0 a 21 y según el rango de la puntuación se puede diferenciar entre caso normal con ausencia de síntomas (de 0 a 7), caso probable (de 8 a 10) o presencia de sintomatología ansiosa o depresiva (11 puntos o más).

En el pre-test del grupo experimental se utilizó una entrevista semi-estructurada de elaboración propia, donde se recogen datos sociodemográficos, información sobre los motivadores y recursos actuales para el desempeño del trabajo, dificultades a las que se enfrentan y los recursos que les faltan o creen que han perdido en su trabajo, información sobre los motivos por los que se demanda el taller psicoterapéutico y sobre los aspectos que consideran necesarios que se aborden y trabajen en el mismo. Así mismo, se solicita información sobre información sobre actividades realizadas en el tiempo libre, características de la actividad profesional e información sobre salud percibida referida al último año, donde se incluyen síntomas psicofisiológicos y conductas asociadas al burnout.

\section{Procedimiento}

El grupo experimental participó en una experiencia psicoterapéutica grupal dirigida al aprendizaje de técnicas para el manejo del estrés laboral, la cual se llevó a cabo de forma intensiva durante tres días en un alojamiento rural (en el Centro de Descan- 
so del "Proyecto Mariposas" de AOEx) ${ }^{1}$ y con una duración de 20 horas efectivas en sesiones de trabajo, siendo en total unas 50 horas de convivencia del grupo.

La iniciativa de esta intervención psicológica grupal surge a raíz de la solicitud y demanda que realiza el personal de enfermería de Hospital de Día del Hospital de Llerena ante la psicooncóloga de AOEx que desempeña allí su labor, para que se lleve a cabo alguna actuación dirigida a disminuir el nivel de estrés que soportan al afrontar día a día su trabajo. La psicooncóloga recoge la demanda del equipo y realiza una propuesta inicial ante la Dirección de Enfermería del Hospital. Posteriormente, se acuerda la realización de una intervención psicoterapéutica grupal en forma de taller en la cual se trabajarían técnicas para el manejo del estrés laboral; asimismo, se acordó que en dicho taller participaría todo el equipo de enfermería de Hospital de Día, que se realizaría durante tres días laborables y que se llevaría a cabo en los alojamientos rurales del "Proyecto Mariposas" de AOEx.

La evaluación pre-tratamiento (T1) del grupo experimental se realizó entre 15 y 20 días previos a la realización de la experiencia psicoterapéutica y de forma individual. Primeramente, se les entregaron los test en un sobre para su cumplimentación. Posteriormente, se concertó con cada uno de los participantes una entrevista individual en la cual, además de darles a conocer los resultados de los tests (HAD y $\mathrm{MBI})$, se les aplicó la entrevista semi-estructurada. Gracias a los datos recopilados en estas entrevistas, pudimos recabar información acerca de las necesidades individuales y grupales del equipo, lo cual nos

1 El "Proyecto Mariposas" de AOEx consiste en la disposición de alojamientos rurales (en el Valle del Ambroz) para pacientes oncológicos con el objetivo de favorecer su bienestar emocional y el afrontamiento de la enfermedad. También pueden ser utilizados para intervenir sobre el burnout de los profesionales. sirvió de guía para estructurar los objetivos y contenidos del programa de tratamiento y las sesiones del mismo. Posteriormente, de acuerdo con dicha programación se presentó a la Dirección de enfermería del Hospital nuestro "Taller de Técnicas para el Manejo del Estrés Laboral". La primera evaluación post-test (T2) se realizó un mes después de la intervención psicoterapéutica grupal. Realizamos una segunda evaluación post-test tras cumplirse un año desde la primera evaluación (T3).

Para conformar el grupo control se solicitó la colaboración al personal de enfermería oncológica de Hospital de Día de otros hospitales pertenecientes a la Comunidad Autónoma de Extremadura. Dicha colaboración fue solicitada a través de las psicooncólogas de AOEx que desempeñan allí su labor, quienes les dieron las explicaciones oportunas sobre el objetivo principal del estudio y les garantizaron la confidencialidad de los resultados. El grupo control realizó una evaluación pre-test (T1) y una sola evaluación post-test (T2) con una diferencia de 30 a 45 días. En las dos evaluaciones las pruebas se entregaban en un sobre el cuál devolvían cerrado y con una clave para identificar la evaluación pre-test y la post-test de la misma persona, al tiempo que se aseguraba su anonimato. Aproximadamente un $40 \%$ accedió a la evaluación y un $80 \%$ completó la misma.

Como objetivo general del Taller de Técnicas para el Manejo del Estrés Laboral nos centramos en proporcionar herramientas para el manejo del estrés que permitan un mejor afrontamiento de las situaciones que producen mayor nivel de desbordamiento personal al profesional de enfermería oncológica. Los objetivos específicos sobre los que se trabajó fueron:

1. Tomar conciencia de las necesidades $y / 0$ dificultades emocionales propias asociadas al trabajo en Hospital de Día. 
2. Aprender a identificar las situaciones que producen mayor nivel de desbordamiento profesional.

3. Desarrollar estrategias de afrontamiento efectivas que permitan manejar las situaciones de estrés laboral de manera que no contribuyan al agotamiento emocional, a la despersonalización y a la falta de realización personal.

4. Mejorar la cohesión grupal, para que a través de la cooperación superen las dificultades que condicionan el trabajo en equipo y se refuerce la autoayuda grupal para prevenir el síndrome de burnout entre sus miembros.

5. Aprender a controlar la sobreactivación psicofisiológica asociada a las situaciones de estrés.

6. Aprender herramientas de comunicación útiles para la relación de ayuda al otro y para el abordaje de situaciones difíciles en el trato con el paciente y con sus familiares.

7. Mejorar las habilidades comunicativas para la resolución de conflictos interpersonales.

8. Aumentar la motivación y la ilusión en el trabajo.

9. Aprender estrategias de afrontamiento para abordar el estrés laboral crónico, en relación a las situaciones que viven los profesionales en su trabajo diario.

El Taller de Técnicas para el Manejo del Estrés Laboral se estructuró según las fases de la intervención para la Inoculación al Estrés de Meichenbaum ${ }^{(23)}$. Estas fueron:

- Fase de conceptualización: es la fase educativa donde se proporciona el marco conceptual. En nuestro Taller se trabajó:

- Naturaleza transaccional del estrés.

- Estrés crónico y desgaste profesional: consecuencias físicas y psíquicas del estrés crónico; relación entre estrés y desgaste profesional.
- Síndrome de Burnout: causas y factores predisponentes; el burnout en profesionales sanitarios; manifestaciones del síndrome (agotamiento emocional, despersonalización y falta de realización personal).

- Estilos de afrontamiento frente al estrés crónico: afrontamiento positivo y negativo del estrés.

- El counselling como herramienta de comunicación en situaciones de ayuda. Actitudes básicas de counselling: empatía, aceptación incondicional y autenticidad.

- Estilos de comunicación. Estilo comunicativo asertivo.

- Fase de adquisición de habilidades y ensayo: es la fase de entrenamiento en técnicas y habilidades de afrontamiento específicas. En nuestro caso se determinaron en:

- Entrenamiento en focalización sensorial y respiración profunda.

- Consejos prácticos para controlar/ prevenir el burnout: cuida el cuerpo, organización del tiempo, estrategias de afrontamiento centradas en el problema, cuidar el estado de ánimo, cuidar la vida social, potenciar el buen humor, estimular la comunicación en el equipo de trabajo, elaborar adecuadamente los duelos, reforzar el sentido positivo de la vida.

- Técnicas y herramientas de counselling: escucha activa, respuestas empáticas, respuestas de validación, preguntas exploratorias, reformulación, persuasión, confrontación, mensajes validantes, mensajes yo, cómo informar, cómo responder a preguntas difíciles, cómo comunicar malas noticias.

- Técnicas de resolución de conflictos: técnica para pedir disculpas, técnica para pedir cambios de conducta a los demás. 
- Entrenamiento en relajación muscular progresiva y visualización guiada.

- Dinámicas de grupo para fomentar la autoestima y la cohesión grupal.

- Fase de aplicación y consolidación: es la fase de puesta en práctica, con aplicación a situaciones y casos similares a los que se enfrentan en su trabajo. Se realizó a través de:

- Role playing de situaciones difíciles con pacientes.

- Ejercicios prácticos para interiorizar herramientas de counselling (con situaciones y diálogos).

- Autorreflexiones para estimular el autoconocimiento de recursos y limitaciones para manejar al paciente hemato-oncológico.

- Análisis de casos para trabajar a nivel emocional la actitud de enfrentarse al sufrimiento y a la muerte de los pacientes.

- Autorreflexiones para estimular el autoconocimiento de recursos y limitaciones que afectan al trabajo en equipo.

- Análisis de situaciones conflictivas en el funcionamiento del grupo en el día a día y aplicación de técnicas de resolución de conflictos.

- Análisis de actitudes positivas para el trabajo en equipo y propuestas de mejora.

- Estrategias de resolución de problemas y situaciones difíciles a través de la cooperación grupal y el apoyo mutuo dentro del equipo.

- Práctica de focalización sensorial y respiración controlada.

- Práctica de relajación muscular progresiva.

- Práctica de visualización guiada.

- Ejercicio físico: paseos al aire libre por el entorno natural.

Hay que señalar que la convivencia se guió y cuidó para estimular la cohesión grupal durante el tiempo de convivencia.
De este modo, la psicooncóloga como terapeuta del Taller programó cuidadosamente la distribución de los miembros del equipo en las tres casas rurales de las que se dispusieron, así mismo realizó una programación en la distribución de tareas (preparación de desayunos, comidas y cenas, con alimentos que ya se llevaron elaborados o semi-elaborados) de manera que cada uno de los miembros del equipo tuviera oportunidad de interactuar con todos los demás miembros de forma colaborativa e igualitaria, en situaciones distintas a las que se producen en su trabajo habitualmente. Igualmente, se cuidó la interacción de forma que enfermeros y auxiliares compartieran casa o tarea en igualdad de rango.

Para los tres día que duró el Taller, se realizó una programación horaria donde se incluía tiempo para las sesiones de trabajo, tiempo para las comidas, tiempo para realizar paseos y tiempo para el descanso. Las sesiones de trabajo psicoterapéutico se realizaron una el primer día de la actividad (en horario de tarde), dos el segundo día (mañana y tarde) y la cuarta el tercer día (por la mañana).

Al comienzo del Taller se acordó entro todos los participantes una serie de normas dirigidas, tanto al cumplimiento de la organización horaria y funcional de la actividad, como al respeto en la interacción entre el grupo de compañeros.

La primera sesión de trabajo se dio comienzo con algunas dinámicas grupales con el objetivo de estimular la motivación y la cohesión grupal, analizando los puntos fuertes del equipo. Después, nos centramos en la conceptualización de la naturaleza transaccional del estrés, el estrés crónico y el desgaste profesional, el síndrome de burnout y los estilos de afrontamiento del estrés. Asimismo, se trabajaron los consejos prácticos para controlar y prevenir el burnout y se realizó un entrenamiento en focalización sensorial y respiración profunda. 
La segunda sesión de trabajo se dedicó, en primer lugar, a la conceptualización del counselling como herramienta de comunicación en situaciones de ayuda, junto con las actitudes básicas asociadas. En cuanto a la adquisición de habilidades, se trabajaron las técnicas y herramientas de counselling de forma intensiva a través de casos, ejercicios prácticos y role-playing. Posteriormente, se realizó un paseo al aire libre, de una hora y media de duración aproximadamente, hasta un paraje natural cercano (Garganta Madrigala de Casas del Monte) donde se puso en práctica la respiración profunda con focalización sensorial.

En la tercera sesión se siguieron trabajando de forma práctica las herramientas y técnicas de counselling y, posteriormente, se enfocó hacia el conocimiento de los estilos de comunicación y el estilo comunicativo asertivo, poniéndose en práctica las técnicas de resolución de conflictos a través de ejercicios prácticos y role-playing. El final de esta sesión de trabajo se dedicó a realizar un entrenamiento en relajación muscular progresiva y en visualización guiada.

La cuarta sesión de trabajo comenzó con un ejercicio de focalización sensorial y respiración profunda. Seguidamente, se realizaron una serie de dinámicas grupales dirigidas a fomentar la autoestima personal y grupal, así como la cohesión del grupo. Para ello se suscitaba a la autorreflexión y al fomento del autoconocimiento de los recursos y las limitaciones personales y grupales que afectan al trabajo en equipo. Se analizaron los puntos débiles del equipo y se realizaron propuestas de mejora, con el compromiso grupal de llevarlas a cabo en la práctica del día a día. Al final de la jornada se realizó un paseo al aire libre de unas dos horas de duración, realizando la ruta senderista desde Segura de Toro hasta el Bosque de Castaños Centenarios del Temblar.
Inmediatamente después de finalizar el Taller, se aplicó una encuesta a los participantes en la que pudieron expresar su grado de satisfacción, en una escala de 1 a 5 (desde "muy poco satisfecho/a" a "muy satisfecho/a), en relación a los siguientes aspectos: contenidos del taller, metodología, aspectos teóricos, aspectos prácticos y aplicabilidad, terapeuta, lugar donde se ha desarrollado y valoración global.

\section{Tratamiento estadístico de los datos}

El tratamiento estadístico de los datos recopilados a través de la evaluación psicológica, se realizó con el programa SPSS para Windows, versión 17.0. Para comparar las medidas entre el grupo experimental y el grupo control utilizamos la Prueba $U$ de Mann-Whitney y para comparar las medidas pre-test y pos-test dentro del mismo grupo utilizamos la Prueba de Wilcoxon de los Rangos con Signo.

Asumimos en la prueba de nuestras hipótesis un nivel de significación inferior a 0,05.

En el caso del grupo experimental al tratarse de comparaciones múltiples (T1T2, T2-T3 y T1-T3), utilizamos la corrección de Bonferroni para controlar la tasa de error. Según este procedimiento, tenemos que basar nuestras decisiones en un nivel de significación correspondiente al alfa tomado y dividido entre el número de comparaciones $(0,05 / 3=0,0167)$.

También realizamos comparaciones entre las distribuciones de frecuencias de los distintos niveles mostrados de las VVDD, utilizando la Prueba Chi-Cuadrado para contrastar el grupo control con el grupo experimental y la Prueba de Homogeneidad Marginal para comprobar los cambios en las distribuciones en el mismo grupo, pero en distintos momentos.

De otra parte, se realizaron pruebas para estimar el tamaño del efecto a través de la diferencia tipificada (d) de Cohen, 
tanto en la comparación de medias de medidas repetidas, como en la comparación de medias entre grupos independientes; en ambos casos, tomamos en cuenta el tamaño del efecto cuando resultó ser grande o moderado $^{(24)}$.

\section{RESULTADOS}

A través de las entrevistas estructuradas e individualizadas realizadas al grupo experimental antes de realizar la experiencia psicoterapéutica, algunos de los participantes expresaron que durante el último año se habían sentido más tristes (33\%), más irascibles (67\%) o más ansiosos (89\%). Un 44\% había recurrido al consumo de ansiolíticos, durante ese mismo período y algunos habían tenido más dis- cusiones con jefes (33\%) o con compañeros $(44 \%)$. En relación a la manifestación de sintomatología, informaron haber experimentado durante ese tiempo dolores de cabeza $(67 \%)$, problemas de sueño $(56 \%)$, trastornos gastrointestinales (44\%) o dolor muscular (78\%).

En las entrevistas todos afirmaron que les gustaba su trabajo y un $66 \%$ hizo referencia a la relación con el paciente como principal incentivo en su labor profesional. La mayoría $(66 \%)$ se refirió a la empatía y a la capacidad de diálogo como los recursos más importantes que le ayudaban a realizar bien su labor. Sin embargo, también comentaron (45\%) que no tenían recursos suficientes para hacer frente a situaciones difíciles con los pacientes o sus familias (p.e. shock emocional o reaccio-

Tabla 1. Medias, desviaciones típicas y comparaciones intergrupales.

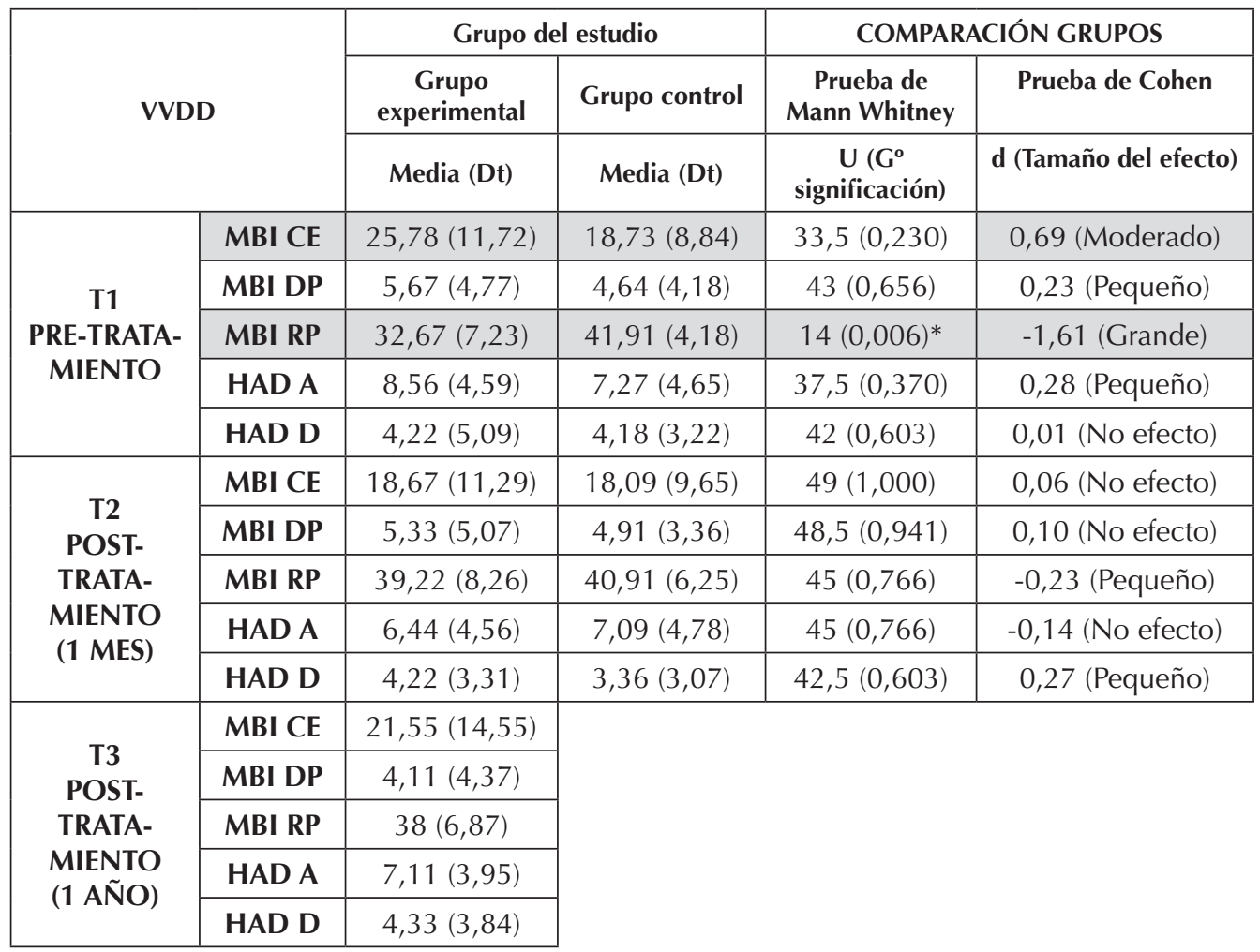

(*) $\alpha<0,05$ 
nes emocionales intensas, preguntas difíciles). Algunos (45\%) comentaban haber perdido con el tiempo la afectividad o la capacidad de implicación emocional con los pacientes, manifestando ante ellos en determinadas ocasiones menos paciencia, cierto desinterés o más distanciamiento.

Según se desprende de los resultados del MBI y del HAD, en el grupo experimental en el momento pre-tratamiento (es decir cuando el equipo de Hospital de día plantea la demanda de tratamiento ante la psicooncóloga), existían niveles de cansancio emocional medios-altos, niveles bajos de realización personal y cierta elevación de la ansiedad. La media del nivel de cansancio emocional (MBI CE) era de 25,78 (Tabla 1), según los puntos de corte empleados esta medida se situaría muy cercana a la frontera entre el nivel medio y el nivel alto de burnout o desgaste profesional. En la Tabla 2 se refleja la alta prevalencia de niveles medios (44,5\%) y de niveles altos $(33,3 \%)$ de burnout en lo referente al cansancio emocional de este grupo. De otra parte, la media de la realización personal (MBI RP) en el grupo antes de la experiencia terapéutica era de 32,67 (Tabla 1), la cual se situaría dentro del nivel indicador de alto burnout. Asimismo, vemos en la Tabla 2, que la mayoría de los integrantes del grupo (un 56\%) presentaban niveles bajos de realización personal, indicadores de alto burnout. Por otro lado, la puntuación media en la Subescala de Ansiedad del HAD en este grupo es de 8,56 (Tabla 1), estando esta puntuación por encima del límite de la ausencia de ansiedad y por debajo del límite de la presencia de la misma. Consultando la Tabla 2 , encontramos que el 55,6\% presenta "ausencia de ansiedad" y el 33,3\% refleja "presencia de ansiedad", estando solo una persona entre las puntuaciones medias. En lo referente a la Subescala de Despersonalización del $\mathrm{MBI}$ y a la Subescala de Depresión del HAD, el grupo experimental refleja puntuaciones medias indicadoras de bajo burnout y ausencia de depresión, respectivamente.

Al comparar los valores medios de las VVDD en el momento pre-tratamiento entre el grupo control y el grupo experimental (Tabla 1), encontramos en este último, puntuaciones superiores en cansancio emocional, en despersonalización y en ansiedad, mientras que la media de la realización personal era más baja en este grupo que en el grupo control. Atendiendo a la comparación estadística de estos datos (Tabla 1), se puso de manifiesto que la diferencia en cansancio emocional, no llegaba a alcanzar la significación estadística en la Prueba de Mann Whitney, pero, según la Prueba de Cohen, el tamaño del efecto era "moderado" (con una diferencia tipificada de 0,69). De otra parte, las diferencias en la Subescala de Realización Personal, establecieron diferencias estadísticamente significativas entre los dos grupos, según la comparación de Mann Whitney y, según la Prueba de Cohen para muestras independientes, un tamaño del efecto grande. Las diferencias entre los dos grupos en cuanto a las puntuaciones de ansiedad en el pre-tratamiento, resultaron no tener valor de significación estadística y el tamaño del efecto resultó ser pequeño (el valor de la diferencia tipificada era de $0,28)$. Por otro lado, al comparar la distribución de frecuencias entre los grupos en los diferentes niveles de las VVDD (Tabla $2)$, nos encontramos nuevamente diferencias estadísticamente significativas en la Subescala de Realización Personal a través de la prueba Chi Cuadrado, ya que el grupo experimental acumula gran parte de la muestra en el nivel indicador de alto burnout, mientras que el grupo control presenta la mayor proporción de sujetos con puntuaciones indicadoras de bajo burnout en dicha Subescala.

En la evaluación post-tratamiento realizada al mes de la experiencia terapéutica 
Tabla 2. Distribución de frecuentas y comparaciones intergrupales.

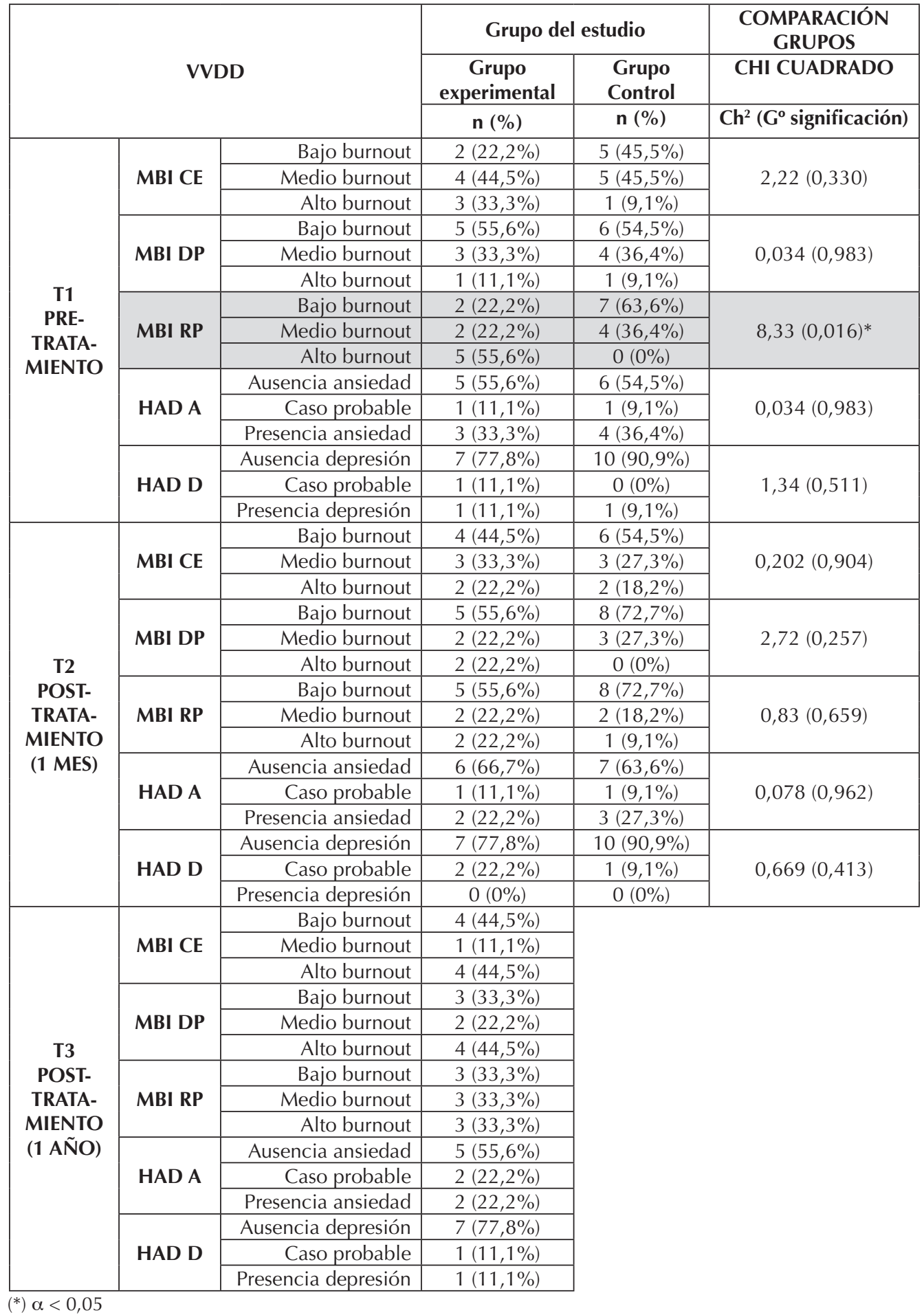


Tabla 3. Comparación intragrupales.

\begin{tabular}{|c|c|c|c|c|}
\hline \multicolumn{5}{|c|}{ COMPARACIÓN MEDIDAS REPETIDAS } \\
\hline \multirow{3}{*}{ VVDD } & \multicolumn{4}{|c|}{ 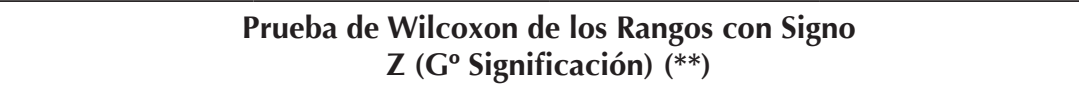 } \\
\hline & \multicolumn{3}{|c|}{ Grupo experimental } & Grupo control \\
\hline & T1 vs T2 & T2 vs T3 & T1 vs T3 & T1 vs T2 \\
\hline MBI CE & $-2,08(0,037)$ & $-1,17(0,235)$ & $-1,36(0,173)$ &,$- 62(0,532)$ \\
\hline MBI DP & $-0,09(0,932)$ & $-1,71(0,088)$ & $-2,23(0,026)$ &,$- 15(0,877)$ \\
\hline MBI RP & $-2,54(0,011)^{* *}$ & $-0,85(0,395)$ & $-2,24(0,025)$ &,$- 77(0,441)$ \\
\hline HAD A & $-2,44(0,015)^{* *}$ & $-0,65(0,518)$ & $-1,16(0,246)$ &,$- 35(0,722)$ \\
\hline HAD D & $-0,14(0,886)$ & $-0,14(0,892)$ & $-0,14(0,888)$ & $-1,03(0,301)$ \\
\hline \multirow{3}{*}{ VVDD } & \multicolumn{4}{|c|}{$\begin{array}{c}\text { Prueba de Cohen } \\
d \text { (Tamaño del efecto) }\end{array}$} \\
\hline & \multicolumn{3}{|c|}{ Grupo experimental } & Grupo control \\
\hline & T1 vs T2 & T2 vs T3 & T1 vs T3 & T1 vs T2 \\
\hline MBI CE & 0,94 (Grande) & -0,34 (Pequeño) & 0,49 (Pequeño) & 0,11 (No efecto) \\
\hline MBI DP & 0,11 (No efecto) & 0,39 (Pequeño) & 0,52 (Moderado) & $-0,11$ (No efecto) \\
\hline MBI RP & $-1,29$ (Grande) & 0,25 (Pequeño) & $-1,15$ (Grande) & 0,29 (Pequeño) \\
\hline HAD A & 0,71 (Moderado) & -0,24 (Pequeño) & 0,52 (Moderado) & 0,06 (No efecto) \\
\hline HAD D & 0 (No efecto) & $-0,05$ (No efecto) & $-0,04$ (No efecto) & 0,40 (Pequeño) \\
\hline \multirow{3}{*}{ VVDD } & \multicolumn{4}{|c|}{$\begin{array}{l}\text { Prueba de Homogeneidad Marginal } \\
\text { Estadístico de HM (G } \text { (G }^{\circ} \text { Significación) }\end{array}$} \\
\hline & \multicolumn{3}{|c|}{ Grupo experimental } & Grupo control \\
\hline & T1 vs T2 & T2 vs T3 & T1 vs T3 & T1 vs T2 \\
\hline MBI CE & $10(0,180)$ & $7(0,317)$ & $7(0,705)$ & $4(1,000)$ \\
\hline MBI DP & $5(0,564)$ & $4(0,102)$ & $4(0,096)$ & $10(0,180)$ \\
\hline MBI RP & $13(0,034) *$ & $10(0,317)$ & $11(0,257)$ & $4(1,000)$ \\
\hline HAD A & $3(0,317)$ & $1(0,317)$ & $40,655)$ & $5(0,157)$ \\
\hline HAD D & $3(0,317)$ & $2(0,317)$ & $0(1,000)$ & $3(0,357)$ \\
\hline
\end{tabular}

$\left.{ }^{*}\right) \alpha<0,05 \quad\left({ }^{* *}\right) \alpha<0,0167$ (Nivel de significación con corrección de Bonferroni)

grupal (T2) que realizamos con el grupo experimental, nos encontramos que las puntuaciones medias en las Subescalas de Cansancio Emocional, de Despersonalización y de Ansiedad habían disminuido respecto al momento pre-tratamiento (T1), al tiempo que la puntuación media de la Subescala de Realización Personal había aumentado (Tabla 1). Por su parte, la puntuación media en la Subescala de Despresión del HAD, no se modificó prácticamente nada entre el pre-test y el post-test del grupo. Como podemos comprobar en la Tabla 3, a través de la Prueba de Wilcoxon de los Rangos con Signo, se produjo una variación estadísticamente significativa en las puntuaciones en lo referente a la realización personal y la ansiedad. La 
Prueba de Cohen para medidas repetidas nos indica que, al mes de la intervención, el tamaño del efecto del tratamiento en este grupo es grande para cansancio emocional y realización personal, y moderado para la ansiedad (Tabla 3).

Por otra parte, la comparación de las distribuciones de frecuencias entre el momento T1 y el T2 en el grupo experimental, que se realizó con la Prueba de Homogeneidad Marginal (Tabla 3), nos refleja una variación con significación estadística en la distribución de los sujetos en la Subescala de Realización Personal, ya que en el momento del pre-tratamiento la mayor acumulación de sujetos se producía en el nivel indicador de alto burnout, mientras que en el post-tratamiento realizado al mes, la mayor parte de la muestra se situaba en el nivel indicador de bajo burnout.

A través de las diferentes comparaciones estadísticas realizadas para las medidas repetidas (Tabla 3 ), se aprecia que en el grupo control o grupo sin tratamiento no se producen variaciones significativas en las distintas medidas tomadas de las VVDD.

Volviendo al grupo experimental y observando las medidas del post-test realizado al año (T3), observamos que las puntuaciones medias de cansancio emocional y de ansiedad aumentan respecto del posttest realizado al mes (T2), mientras que las medias de despersonalización y de realización personal disminuyen durante el mismo período. La puntuación en depresión permanece poco variable. Todas estas diferencias resultaron tener un tamaño del efecto pequeño (Prueba de Cohen, Tabla 3). La Prueba de Wilcoxon y la Prueba de Homogeneidad Marginal, no identificaron diferencias significativas en las variables ni en las puntuaciones medias, ni en las distribuciones, respectivamente.

Si nos centramos en las diferencias existentes en el grupo experimental entre el momento pre-tratamiento (T1) y el post-tratamiento realizado al año (T3), observamos que las puntuaciones medias de cansancio emocional, de despersonalización y de ansiedad disminuyen, mientras que la puntuación de realización personal aumenta y la de depresión no varía. Las comparaciones estadísticas de los valores de la VVDD tomados en esos dos momentos, a través de la Prueba de Wilcoxon de los Rangos con Signo nos indica valores importantes para la despersonalización $(Z=-2,23 ; p=0,026)$ y para la realización personal $(Z=-2,24 ; p=0,025)$. Sin embargo no alcanzan el valor de significación estadística, ya que en este caso hay que aplicar la corrección de Bonferroni, debido a que se trata de comparaciones múltiples y tomar como nivel de significación un alfa inferior a 0,016. No obstante, la Prueba de Cohen para muestras relacionadas nos indica un tamaño del efecto moderado para la despersonalización y la ansiedad y un efecto grande para la realización personal. La comparación de distribución de frecuencias en los distintos niveles de los valores de las VVDD entre T1 y T3 (a través de la Prueba de Homogeneidad Marginal), no detecta cambios con valor de significación estadística en dichas distribuciones (Tabla 3).

\section{DISCUSIÓN}

En la situación de partida, nos encontramos con un equipo de enfermería oncológica (el de Hospital de Día del Hospital de Llerena, perteneciente al Servicio Extremeño de Salud -SES-) que demanda una intervención psicoterapéutica grupal, siendo conscientes de las repercusiones emocionales que las circunstancias de su trabajo tienen a nivel individual y a nivel de equipo. La evaluación inicial nos pone de manifiesto la existencia de niveles elevados de cansancio emocional, de niveles bajos de realización personal, así como de cierta elevación de la ansiedad (Tabla 
1). Un $67 \%$ de los miembros del equipo manifestaba niveles elevados en, al menos, uno de los tres indicadores de burnout y el $44 \%$ presentaba dos o tres de los indicadores al mismo tiempo, siendo los más frecuentes el cansancio emocional y la falta de realización personal.

El grupo control se formó con profesionales de diferentes Unidades de Hospital de Día (de varios hospitales del SES), que realizan labores similares a las de nuestro grupo de tratamiento, y en quienes también se deja sentir la fatiga de compasión; así, por ejemplo, observamos que un $46 \%$ presentaba niveles medios de cansancio emocional. Sin embargo, existen diferencias importantes en el pre-test en comparación con el grupo experimental (Tabla 1), por cuanto los sujetos del grupo control tienen menos cansancio emocional (con una diferencia moderada) y más realización personal (con una diferencia estadísticamente significativa y un tamaño del efecto grande). En el grupo control el 92\% de los sujetos no presentaba elevados ninguno de los indicadores de burnout y solo un $18 \%$ manifestaba alto uno de ellos en la evaluación pre-tratamiento.

Podemos decir, con bastante seguridad, que nuestro equipo de enfermería del grupo experimental manifestaba en el pre-tratamiento un nivel más elevado de desgaste profesional. Las manifestaciones características de este desgaste se dejaban sentir a nivel emocional, a nivel fisiológico y a nivel relacional. De este modo, la mayoría de estos profesionales se quejaban de un aumento importante durante el último año de ansiedad y de irascibilidad, asociado a un incremento de roces con compañeros o con jefes, con presencia de diferentes problemas fisiológicos, especialmente problemas de sueño y dolores musculares o de cabeza. Estos problemas fueron manifestados a través de las entrevistas semiestructuradas individuales realizadas antes del tratamiento.
A través de la información recopilada en la evaluación pre-tratamiento del grupo experimental, realizamos un análisis funcional de la conducta, que nos puede ayudar a comprender mejor los factores antecedentes y consecuentes asociados al burnout o desgaste en estos profesionales. Como veremos a continuación, son de especial relevancia en este análisis las reacciones de estrés frente a situaciones difíciles con pacientes que implican una alta demanda emocional, debido a las consecuencias que tienen a largo plazo desde el punto de vista emocional, y que inciden de modo directo en las conductas asociadas al desgaste profesional.

Como antecedentes distales podemos destacar tres tipos de factores precursores de las conductas problemas asociadas al estrés y al desgaste profesional manifestadas por el equipo del Hospital de Día tratado. Uno de ellos se refiere a las propias condiciones estructurales del trabajo que se desempeña en Hospital de Día, donde se incluye la sobrecarga de trabajo, la presión temporal, los riesgos en las pruebas y tratamientos que aplican. El segundo tipo de precursores se refiere a factores interpersonales, entre los que cabe destacar primeramente la continua carga emocional a la que están sometidos los profesionales de oncología y que se asocia al contacto con pacientes que viven situaciones de alto nivel de sufrimiento. Otro factor interpersonal se refiere a los déficits de comunicación interna del equipo, que dificultan el abordaje de situaciones difíciles de trabajo, comprometiendo la ayuda mutua o complicando la cohesión grupal. El tercer grupo de antecedentes distales se refiere a factores de tipo personal asociados al tipo de estrategias de afrontamiento utilizadas ante el estrés y la carencia de formación específica en habilidades y técnicas comunicativas aplicables en la relación de ayuda a pacientes y familiares en situaciones de alta demanda emocional. 
Entre los antecedentes proximales o disparadores del estrés destacamos aquellas situaciones en que se producen demandas emocionales de pacientes o de familiares, situaciones donde los pacientes sufren crisis de ansiedad y/o derrumbes emocionales, donde tienen la necesidad de desahogo emocional, o cuando manifiestan una reacción psicológica intensa ante recaídas o malas noticias, así como cuando plantean preguntas difíciles. Otras situaciones con alta demanda emocional que suponen una activación inmediata del estrés de los profesionales de oncología de nuestro grupo de tratamiento son el shock anafiláctico o el éxitus en la Sala de Tratamientos, o el hecho de saber que un paciente oncológico está ingresado en planta en situación terminal o agonizante, especialmente aquellos que han estado varios años en tratamiento o con los que han establecido una relación especial (en ocasiones con sobreimplicación emocional).

Como conductas problema asociadas al estés en nuestro grupo de tratamiento, identificamos en al área cognitiva el balance negativo entre demandas percibidas $y$ competencias percibidas ante las situaciones anteriormente descritas, con sensación de sobreexigencia, junto con expectativas negativas sobre la competencia comunicativa y la eficacia personal ante dichas situaciones desbordantes. En el área fisiológica y emocional destacamos la sensación de amenaza, de falta de control, de indefensión, así como sobrecarga emocional, sobreactivación, ansiedad e irascibilidad. Desde el punto de vista comportamental, los profesionales del grupo experimental evaluados ante estas situaciones desbordantes a veces suelen expresar gestos $y$ verbalizaciones que establecen una cierta distancia emocional ante los pacientes, inhibiendo la comunicación y la profundización en el tema que el paciente desea/ necesita desahogar, por miedo a llegar a una situación de difícil manejo emocional.
En otras ocasiones se produce una sobreimplicación emocional con los pacientes o los familiares, sin una adecuación en el límite de lo personal y lo profesional, que llega a producir una gran fatiga de compasión.

El temor a la sobreimplicación emocional o a no dar una respuesta adecuada, les lleva en ocasiones a evitar situaciones difíciles, a distanciarse emocionalmente del paciente y a no responder a las demandas emocionales de los mismos, con lo cual a veces "se llevan el paciente a casa", es decir que, ante la disonancia cognitiva que les produce no dar una respuesta adecuada a la demanda emocional planteada, siguen pensando en el paciente una y otra vez fuera de su horario de trabajo.

El distanciamiento emocional puede suponer un alivio de la tensión emocional en el momento, a corto plazo, pero a largo plazo genera más malestar, aumentando el cansancio emocional y la falta de realización personal, y disminuyendo la autoestima, la motivación y la ilusión en su labor profesional.

La falta de un espacio y un tiempo específico para ello, hace que a menudo no se hable con los compañeros de lo que les sucede, sin facilitarse con ello el desahogo emocional, ni permitiéndose la ayuda mutua o la elaboración de duelos. De otro lado, la sobreactivación y sobrecarga emocional como generadores de irascibilidad, sirven de detonante para que aumenten los roces y conflictos en el día a día, con lo que se produce una mayor debilidad en la cohesión grupal. Todo ello puede contribuir a que el apoyo social y emocional dentro del equipo disminuya a lo largo del tiempo, lo cual hace más duro el afrontamiento de las situaciones de estrés que se viven en el trabajo.

En base a todo lo anterior, nos planteamos realizar una intervención psicológica grupal, ya que la demanda había sido por parte de todo el equipo, con un enfoque 
flexible y donde se trabajaran un conjunto de herramientas terapéuticas que permitieran abordar la amplitud de la problemática planteada. De este modo, nos planteamos el trabajo psicoterapéutico desde el esquema de la intervención para la Inoculación del Estrés (ver apartado de Procedimiento), utilizando un programa multicomponente.

Consideramos importante para la motivación y la implicación personal de los participantes, que el Taller de Técnicas para el Manejo del Estrés Laboral, se realizara en días y horarios laborables, como parte de su trabajo y sin que resultara ser un estresor añadido en su vida cotidiana. La cooperación por parte de Dirección de Enfermería del Hospital, resultó clave en este sentido y aportó al equipo la sensación de que sus quejas se sentían oídas y atendidas. De otra parte, la posibilidad de realizar nuestro Taller en un alojamiento rural (ver Procedimiento), hizo que nos planteáramos el beneficio que podía tener para el equipo la convivencia fuera del entorno habitual de trabajo como forma de reforzar la cohesión grupal. De este modo, como se comenta en el Procedimiento, se programaron previamente el reparto de habitaciones y el reparto de tareas con el objetivo de guiar y cuidar la interacción de forma colaborativa e igualitaria.

De otra parte, consideramos que podría ser relevante, de cara a obtener resultados óptimos, que la experiencia psicoterapéutica se realizara en un entorno natural atractivo, con un potencial relajante y desestresante $^{2}$, donde fuera posible incluir paseos al aire libre como parte de los recursos terapéuticos contra el estrés. Decir también, que se cuidó el cumplimiento de la programación horaria y las normas de convivencia acordadas con el grupo, para

2 Estudios preliminares sobre el Proyecto Mariposas de AOEx se pone de manifiesto el beneficio emocional sobre los pacientes, con reducción de la ansiedad y mejora de la salud percibida y de la sensación de calidad de vida. la consecución de los objetivos terapéuticos planteados.

El Taller se llevó a cabo según lo previsto. Se fueron utilizando y desplegando las diferentes herramientas psicoeducativas y psicoterapéuticas a lo largo de cuatro sesiones de trabajo de cinco horas de duración cada una, y a lo largo de 50 horas de convivencia. Nos encontramos con un grupo motivado, que mostró un gran interés y una gran apertura ante todas las propuestas de trabajo.

Como se comenta en el Procedimiento, tras la conclusión de la experiencia terapéutica, se administró a los participantes un cuestionario anónimo para valorar la satisfacción en relación a los diferentes aspectos del taller (contenidos, metodología, aplicabilidad, terapeuta, ubicación y valoración global), reflejando entre el $90 \%$ y el $100 \%$ sentirse "muy satisfechos" en relación a los mismos (todas las puntuaciones se encontraban entre "satisfecho/a" y "muy satisfecho/a").

En la evaluación realizada un mes después del tratamiento en el grupo experimental, nos encontramos una normalización y equiparación de las medidas de las VVDD en relación al post-test grupo control (Tablas 1 y 2 ). Mientras que en el grupo control no hay cambios significativos en los parámetros emocionales evaluados entre la primera y la segunda evaluación, sí se producen cambios en el grupo experimental entre la evaluación pre-tratamiento y la post-tratamiento, pudiendo llegar a afirmar que la intervención psicoterapéutica grupal Ilevada a cabo en el grupo mejoró los niveles de cansancio emocional, ansiedad y realización personal (Tabla 3), contribuyendo con ello a una disminución del grado de desgaste profesional y del estrés.

Entre la evaluación post-tratamiento llevada a cabo al mes de la intervención psicológica (T2) y la realizada al año del pre-test (T3) no se producen cambios esta- 
dísticamente significativos ni en los valores ni en los niveles de las VVDD (Tabla 3), solo podemos hablar de pequeños efectos en algunas de las variables producidos en este período. Dichos pequeños cambios se refieren a un ligero aumento en cansancio emocional y en ansiedad, con cierta disminución de la realización personal, pero también hablamos de una pequeña disminución en el factor despersonalización. En función de estos resultados podemos decir que pasado un año de la intervención psicológica algunos aspectos relacionados con el desgaste profesional empeoran ligeramente $y$, sin embargo, otro factor emocional del burnout, como es la despersonalización, en su caso mejora ligeramente.

En la Tabla 3, podemos ver cómo entre el pre-test (T1) y el último post-test del grupo de tratamiento (T3) no hay diferencias estadísticamente significativas en los valores de las VVDD, por cuanto la corrección de Bonferroni realizada a la Prueba de Wilcoxon nos obliga a considerar un nivel de significación muy exigente en el contraste de nuestra hipótesis. Pero si atendemos al tamaño del efecto del tratamiento ( $d$ de Cohen), sí encontramos cambios de tamaño moderado y grande en algunos de los parámetros, que nos harían pensar en la efectividad de la intervención psicológica transcurrido ese tiempo, en concreto en lo referente a la bajada de la ansiedad y la despersonalización y al aumento de la realización personal.

Analizando variable por variable y en relación a los factores emocionales del burnout, se pone de manifiesto, en primer lugar que el cansancio emocional disminuye tras la intervención psicológica, pero aumenta después de un año, aunque no llega al mismo nivel inicial. En segundo lugar, la despersonalización inicialmente disminuye levemente y al año vemos que se distancia aún más del valor inicial. Y por último, en lo que respecta a la realización personal, que recordamos que era el factor que marcaba más diferencias con el grupo control en el pre-test, aumenta de forma significativa inmediatamente tras el tratamiento, permaneciendo ese cambio bastante estable con el transcurso del tiempo. Si tratamos de dar explicación a estos cambios, diríamos primeramente que el tratamiento resultó inicialmente muy efectivo para la disminución del cansancio emocional porque se trabajaron técnicas dirigidas a contrarrestar las sensaciones de fatiga, agotamiento o frustración en el trabajo. No obstante, las situaciones de interacción con los pacientes en Hospital de Día producen una continua fatiga de compasión que ha podido producir a lo largo de los meses una nueva elevación en el parámetro. De otra parte, creemos que el intenso trabajo en actitudes y herramientas básicas de counselling, que llevamos a cabo en la intervención, produjeron un efecto positivo y duradero sobre el parámetro de realización personal. Este factor tiene mucha relación con la forma de acercamiento y apertura hacia los pacientes, con la sensación de ser útil, con la idea de poder influir positivamente, con la percepción de autoeficacia en el afrontamiento de los problemas planteados por los pacientes y con la sensación de satisfacción por trabajar con ellos. Por otro lado, el hecho de que la despersonalización haya disminuido a largo plazo, puede indicarnos que sea un factor que necesite más tiempo de evolución, para que se interioricen adecuadamente las herramientas de comunicación y puedan madurar ciertas actitudes de sensibilidad o interés hacia los pacientes.

Respecto a la ansiedad, nuestros datos indican un efecto positivo y significativo del tratamiento psicoterapéutico en un primer momento (al mes), produciéndose posteriormente (al año) un ligero repunte, por lo que el efecto del tratamiento es bastante moderado con el paso de los meses. Podemos afirmar que las téc- 
nicas cognitivo-conductuales utilizadas para modificar el estilo de afrontamiento frente al estrés dieron lugar a un cambio positivo a corto plazo muy importante $y$ que, probablemente, se necesite realizar alguna sesión de reforzamiento con el grupo para que sigan utilizando las técnicas aprendidas (por ejemplo técnicas de relajación).

Por último, las medidas de depresión nos indican pocos cambios y poco efecto a nivel grupal, si bien es cierto que los valores inicialmente ya eran bajos y similares al grupo control.

Debido a las observaciones realizadas anteriormente en relación al cansancio emocional y a la ansiedad, consideramos importante plantearse la posibilidad de realizar una nueva intervención psicológica grupal, que refuerce las herramientas aprendidas y permita el aprendizaje de técnicas específicas para el abordaje de la fatiga de compasión (p.e. técnicas de mindfulness).

Todas estas afirmaciones referidas a nuestros datos debemos realizarlas con la debida cautela, ya que estamos ante un estudio piloto y somos conscientes del pequeño número de sujetos que componen nuestros grupos estudiados, lo cual condiciona la validez de los resultados.

De otra parte, hay que comentar que son escasos los trabajos empíricos cuyo objetivo sea valorar la efectividad de intervenciones terapéuticas realizadas con personal sanitario de oncología para el control del burnout, apuntando los que están publicados hacia la efectividad de los programas multicomponente $\mathrm{i}^{(16,17)}$.

Existen experiencias interesantes como la de Chacón et $\mathrm{al}^{(25)}$ quienes realizaron una serie de talleres psicoeducativos a lo largo de 16 sesiones de 1-2 horas de duración cada una, con enfermeros de oncología, trabajando técnicas para la mejora del afrontamiento eficaz ante los estresores laborales y obteniendo resul- tados muy positivos en lo relativo a la disminución de los niveles de burnout. Por su parte, Cruzado ${ }^{(26)}$ obtiene cambios clínicamente significativos a los tres meses de la intervención psicoterapéutica en el $50 \%$ de los miembros del equipo de oncología radioterápica tratado en lo relativo al cansancio emocional y la despersonalización. En este caso, se llevó a cabo un completo trabajo terapéutico a lo largo de seis sesiones, donde se incluía psicoeducación, estrategias de control del estrés, pautas para el autocuidado, entrenamiento en respiración, reestructuración cognitiva, trabajo en grupo y apoyo mutuo, así como, manejo de situaciones difíciles en la interacción con pacientes. Otro trabajo de gran interés es el de Le Blanc et $\mathrm{al}^{(27)}$, quienes presentan un estudio cuasi-experimental muy adecuado en cuanto a la rigurosidad y al número de sujetos empleados, en donde la intervención psicoterapéutica fue realizada a lo largo de seis sesiones mensuales de tres horas de duración cada una. Estos autores observaron una disminución significativa en el nivel de cansancio emocional al concluir la experiencia de tratamiento y a los seis meses de la finalización de la misma. También encontraron menos despersonalización al acabar el tratamiento pero no en el seguimiento. Salvando las distancias en cuanto al tamaño y al diseño con este último estudio mencionado, nuestro trabajo parece incidir sobre un mayor número de aspectos emocionales del desgaste profesional, lo cual puede deberse bien, al tipo de herramientas terapéuticas utilizadas en cada estudio, bien al efecto de la convivencia en un entorno natural, o bien a un efecto combinado de ambos factores.

Por último, hay que decir que no hemos encontrado ningún estudio publicado cuya intervención psicológica grupal fuera similar a la nuestra, en lo relativo a la realización de forma intensiva del tra- 
bajo terapéutico, creando un entorno de convivencia para el grupo y aprovechando las ventajas que ofrece un entorno natural rural, por lo que creemos que esta nueva línea de investigación podría trabajarse más en el futuro.

\section{CONCLUSIONES}

Nuestros resultados apoyan solo parcialmente la hipótesis de trabajo, ya que el efecto del tratamiento no se produce por igual en todos los parámetros emocionales evaluados, así mismo dicho efecto es distinto al mes y al año de la experiencia.

Según nuestro trabajo, en el grupo de tratamiento, transcurrido un mes de la intervención psicoterapéutica grupal, se había producido una mejoría significativa de la ansiedad y de la realización personal, igualmente se produjo en este período un gran efecto positivo sobre el cansancio emocional. Estos cambios no produjeron diferencias respecto al grupo control en el post-tratamiento, sino más bien dieron lugar a una equiparación entre los grupos en los valores de las variables dependientes consideradas, en comparación con la situación pre-tratamiento. Esto es explicable teniendo en cuenta que en la situación de partida, el grupo de tratamiento había demandado la intervención psicológica, por cuanto manifestaba mayores signos de desgaste profesional, principalmente en lo referente a la realización personal (también había un efecto diferencial moderado en relación al cansancio emocional).

Tras casi un año de la intervención psicológica, sigue habiendo un efecto positivo importante sobre la realización personal y la despersonalización, aunque los efectos sobre el cansancio emocional y la ansiedad se han ido debilitando. Esto nos puede estar indicando, la necesidad de realizar intervenciones terapéuticas sistematizadas en el tiempo (talleres con periodicidad anual) que contribuyan, al reforzamiento de las herramientas y habilidades trabajadas con anterioridad o a la adquisición de otras nuevas.

No podemos atrevernos a generalizar resultados y debemos realizar afirmaciones cautelosas, ya que es importante seguir investigando sobre este tipo de intervenciones y utilizar muestras más numerosas. No obstante, creemos que nuestro trabajo psicoterapéutico grupal resultó efectivo y positivo para nuestro equipo de Hospital de Día tratado, produciendo efectos positivos importantes sobre los niveles de desgaste profesional que inicialmente presentaban, especialmente a corto plazo en cuanto al cansancio emocional, la ansiedad y la realización personal y, a largo plazo, en lo referente a la despersonalización y la realización personal.

Creemos, por tanto, que sería necesario estudiar más a fondo el proceso de intervención que llevamos a cabo, ya que a la estructura de un paquete terapéutico para la inoculación al estrés, le añadimos el componente de convivencia grupal intensiva y el componente de la vivencia en un entorno natural rural. Saber cuánto y cuáles de los cambios producidos en el grupo se deben a cada uno de estos componentes, nos ayudaría a plantear en el futuro intervenciones más efectivas y eficaces.

Por último, queremos destacar la importante labor que realiza o puede realizar el/la psicooncólo/a, como parte integral esencial en los equipos sanitarios de oncología, en lo referente al abordaje preventivo o terapéutico del estrés laboral y del desgaste profesional de sus miembros, fomentando con ello la salud psicofisiológica de los profesionales, la cohesión y rendimiento del equipo, así como la mejora de la calidad asistencial que se proporciona al paciente y su familia. 


\section{REFERENCIAS BIBLIOGRÁFICAS}

1. Maslach C, Jackson S. MBI: Maslach Burnout Inventory. Palo Alto: Consulting Psychologists Press; 1986.

2. Maslach C, Leiter MP. The truth about burnout: How organizations cause personal stress and what to do about it. San Francisco: Jossey-Bass, 1997.

3. Pines A, Aronson E, Kafry D. Burnout: From tedium to personal growth. New York: Free Press, 1981.

4. Odoñez A. Causas del síndrome de agotamiento profesional. En: González-Barón M, Lacasta MA, Ordóñez A, editores. El síndrome de agotamiento profesional en oncología. Madrid: Editorial Médica Panamericana, 2008. p. 9-17.

5. Maslach C. Burnout: A social psychological analysis. En: Sander G, Suls J, editores. The burnout syndrome. Ridge: London House, 1982.

6. Leiter MP, Maslach C. Banishing burnout: Six strategies for improving your relationship with work. San Francisco: JosseyBass, 2005.

7. Limonero JT, Fernández-Castro J. Etiología. Del estrés al "burnout" un modelo etiológico del Síndrome de quemarse en el trabajo. En: González-Barón M, Lacasta MA, Ordóñez A, editores. El síndrome de agotamiento profesional en oncología. Madrid: Editorial Médica Panamericana; 2008. p. 19-33.

8. Gil-Monte PR, Peiró JM. Perspectivas teóricas y modelos interpretativos para el estudio del síndrome de quemarse por el trabajo. Anal Psicol 1999; 15: 261-8.

9. Grau A, Suñer R, García, MM. Desgaste profesional en el personal sanitario y su relación con los factores personales y ambientales. Gac Sanit 2005; 19 (6): 63-70. Doi:10.1016/S02139111(05)71397-2

10. Demeuroti E, Bakker AR, Nachreiner F, Schaufeli WB. A model of Burnout and life satisfaction among nurses. J Adv Nurs.
2000; 32: 454-64. Doi:10.1046/j.13652648.2000.01361.x

11. Sosa EN. Frecuencia de los síntomas del síndrome de Burnout en profesionales médicos. Rev Med Rosario. 2007; 73(1): 12-20.

12. Moreno-Jiménez B, Garrosa E, Morante ME. El desgaste profesional o Burnout en los profesionales de oncología. Bol Psicol 2003; 79: 7-20.

13. Albaladejo R, Villanueva R, Ortega P, Astasio P, Calle ME, Domínguez V. Síndrome de Burnout en personal de enfermería de un Hospital de Madrid. Rev Esp Salud Publica. 2004; 78 (4): 505-16. Doi:10.1590/ S1135-57272004000400008

14. Cibanal L, Arce MC, Carballal MC. Técnicas de comunicación y relación de ayuda en Ciencias de la Salud. Barcelona: Elsevier, 2010.

15. Cruzado JA. Tratamiento psicológico en pacientes con cáncer. Madrid: Síntesis, 2010.

16. Cancio H, García H. "Burnout" en oncología - tratamiento. En: González-Barón M, Lacasta MA, Ordóñez A, editores. El síndrome de agotamiento profesional en oncología. Madrid: Editorial Médica Panamericana, 2008. p. 61-74.

17. Turner J, Kelly B, Girgis A. Supporting oncology health professionals: A review. Psychooncology 2011; 5: 77-82. Doi:10.1007/s11839-011-0320-8

18. Seisdedos N. Manual MBI, Inventario de Burnout de Maslach. Madrid: TEA; 1997.

19. Moreno B, Oliver C, Aragoneses A. El "burnout", una forma específica de estrés laboral. En: Buela-Casal G, Caballo VE, editores. Manual de psicología clínica aplicada. Madrid: Siglo XXI, 1993. p. 271-84.

20. Redondo A, Gómez C, Moreno V. Evaluación del síndrome de agotamiento profesional. En: González-Barón M, Lacasta MA, Ordóñez A, editores. El síndrome de agotamiento profesional en oncología. Madrid: Editorial Médica Panamericana, 2008. p. 45-52. 
21. Zigmond AS, Snaith RP. The Hospital Anxiety and Depressión Scale. Acta Psychiatr Scand 1983; 67: 36170. Doi:10.1111/j.1600-0447.1983. tb09716.x

22. Caro I, Ibáñez E. La Escala Hospitalaria de Ansiedad y Depresión. Su utilidad en Psicología de la Salud. Bol Psicol 1992; 36:43-69.

23. Meichenbaum D. Manual de inoculación de estrés. Barcelona: Martínez Roca; 1987.

24. Morales-Vallejo P. El tamaño del efecto (effect size): análisis complementarios al contraste de medias. Madrid: Universidad Pontificia de Comillas, 2010.

25. Chacon M, Grau J, Massip C, Infante, I, Grau R, Abadal Y. El control del syndrome de desgaste professional o burnout en enfermería oncológica. Una experiencia de intervención. Ter Psicol. 2006; 24(1): 39-53.

26. Cruzado-Rodríguez JA, Pérez-Serrano AM, Mateo-Ortega D, Cabo-Domínguez, R, Amurrio-López, LM. Casos Clínicos. En: González-Barón M, Lacasta MA, Ordóñez A, editores. El Síndrome de Agotamiento Profesional en Oncología. Madrid: Editorial Médica Panamericana, 2008. p. 89-132.

27. Le Blanc PM, Hox JJ, Schaufeli WB, Taris TW, Peeters MC. Take care! The evaluation of a team-based burnout intervention program for oncology care providers. J Appl Psychol 2007; 92(1): 213-27. Doi:10.1037/0021-9010.92.1.213 